\title{
Strategy Roadmaps: New Forms, New Practices
}

\author{
Alan F. Blackwell ${ }^{1}$, Rob Phaal ${ }^{2}$, Martin Eppler ${ }^{3}$, and Nathan Crilly ${ }^{4}$ \\ ${ }^{1}$ University of Cambridge Computer Laboratory, Cambridge CB3 0FD UK, ${ }^{2}$ Institute for \\ Manufacturing, Cambridge, ${ }^{3}$ University of Lugano, ${ }^{4}$ Engineering Design Centre, Cambridge , \\ \{afb21,rp108,nc266\}@cam.ac.uk, martin.eppler@lu.unisi.ch
}

\begin{abstract}
Strategy roadmaps are a class of abstract visual representation that is unusually diverse in diagrammatic style. A large corpus of roadmap visualisations was collected, and analysed using a collaborative consultation method involving expert participants. The results indicate organizing principles for the broader study of diagram use within strategic management. They also provide an opportunity for some basic design advice that can be given to professional strategy practitioners or managers who need to create diagrams of this kind. Finally, this novel context of diagram use has required the development of new research methods that may be of value in other Diagrams research.
\end{abstract}

\section{Introduction}

In this paper we describe and analyse a class of diagrams that is defined, not by specific visual characteristics and syntax, but by the particular role that it plays within social and organisational contexts. Strategy roadmaps, as described below, include a rather diverse range of diagrammatic representations, all of which can be used to organise and communicate information related to plans for the future. Whereas much research in diagrammatic reasoning starts from an agreed visual convention for a new (or old) type of diagram, the field of strategy roadmaps is different. Indeed, strategy roadmaps are especially interesting because the conventions that support their usage are still in the process of being discovered and negotiated.

The very phrase "strategy roadmap" offers those who might create them (e.g. company directors, management consultants, public policy experts) an apparently intuitive impression of why its construction might be undertaken, how a suitable visual representation might be formed, and what its uses might be. However, there is no simple agreement among strategy practitioners on any of these issues, and they have consequently been lured into the invention of a wide range of different diagrammatic structures.

In the absence of established and consensual best practice, those creating strategy roadmaps have collectively generated a large body of diagrams that are highly heterogeneous in form whilst being highly homogeneous in function. This combination of diversity, continuity and quantity provides us with two distinct yet complementary research opportunities. Firstly, with respect to academic diagram theory, strategy roadmaps provide us with an unusually large pool of visualizations that require new research methods, as described later, that are likely to be of more general utility in Diagrams research. Secondly, with respect to professional practice, strategy roadmap- 
pers form a community that develop representations but operate largely without the support of relevant diagrammatic knowledge. This offers potential for diagrams researchers to apply their research in a beneficial way. To address both these opportunities we designed and conducted a research study that both provides new methods and opportunities for diagrams research, and also practical guidance for the roadmapping community.

In this paper we first offer a brief history of the development of strategy roadmapping. We then describe a research corpus that captures the diversity of diagrams developed for roadmapping. We propose a research method that investigates this diversity by taking into account both the communicative intentions of the diagram creators and the effective design options available to them. Finally, we present results from a research project applying that methodology, including both analytic findings, and an experimental application of the findings.

\section{Roadmapping - visualizing strategy}

The 'technology roadmapping' approach was originally developed by Motorola in the 1970s to support improved alignment between technology and product development (Willyard \& McClees, 1987). A key feature of this process was the synthesis of the main elements of the strategic plan into a simple high-level visual representation (see Fig. 1). Bob Galvin, who was CEO of Motorola during the period when roadmapping was established, provides the following definition (Galvin, 1998): “A 'roadmap' is an extended look at the future of a chosen field of inquiry composed of the collective knowledge and imagination of the brightest drivers of change in that field".

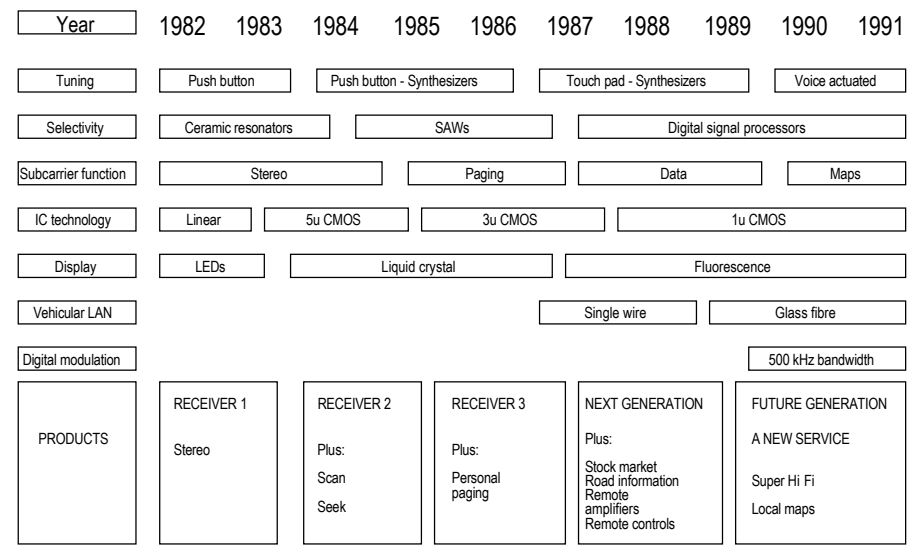

Fig. 1. Motorola roadmap (Willyard \& McClees, 1987)

Following on from Motorola, the roadmapping approach has been adopted widely by many organisations around the world, at company, sector and national levels (for example, Cosner et al., 2007; de Laat \& McKibben, 2003). The underlying concepts are very flexible, and roadmapping methods have been adapted to suit many different 
goals, supporting innovation, strategy and policy development and deployment (Phaal et al., 2004). Subsequent widespread adoption of the method can be readily demonstrated through a search of the Internet. A survey of public-domain roadmap documents by the second author (Phaal, 2006) identified more than 900 examples from a wide range of sectors, including energy, transport, aerospace, electronics, ICT, manufacturing, construction, healthcare, defence, materials and science.

The types of issues that stimulate organisations to deploy roadmapping methods tend to share some characteristics that are inherently challenging for managers and policy makers to deal with: 1) the high level of complexity of the systems being considered; 2) the high level of uncertainty related to the forecasts and assumptions that have to be made; and 3) the high level of ambiguity associated with the many different stakeholder views involved in roadmap development.

The most frequently cited benefit of the roadmapping approach is that of communication, enabled primarily by the visual roadmap formats used. The process of roadmap development brings together the various key stakeholders and perspectives needed to develop understanding of complex systems and issues, building consensus about the way forward. Once a roadmap has been developed it can be more widely disseminated, acting as a reference point for ongoing dialogue and action. Design principles that support these kinds of activity have been described, in a more generic sense, by Bresciani et al. (2008a, 2008b). Nevertheless, although visual representation of strategy lies at the heart of strategy roadmapping, there has been little research directed towards understanding the visual function and structure of roadmaps.

\section{The Research Corpus}

In order to address this research question, the second author engaged in more detailed analysis of the 900 documents identified in his survey. Of these, approximately 450 were constructed around a central visual representation (many documents described as "roadmaps" simply describe strategy in a text format). These 450 figures were extracted into a corpus including: an A5 scaled version of each figure, the original caption for the figure, and a reference to the source document. Initial examination of this rich corpus revealed that many different formats had been developed for representing strategy, as illustrated in Fig. 2. This diversity demonstrated the research opportunity arising from the strategy roadmap, as a diagrammatic application in which there is not yet an agreed set of visual conventions. It also highlighted a professional need to understand the visual dimensions of roadmapping better - what are the principles for 'good' roadmap design? The motivation and challenge for the main part of our research was to develop a method of characterizing the corpus in ways that would be relevant to the practitioner community from which it had been collected. 

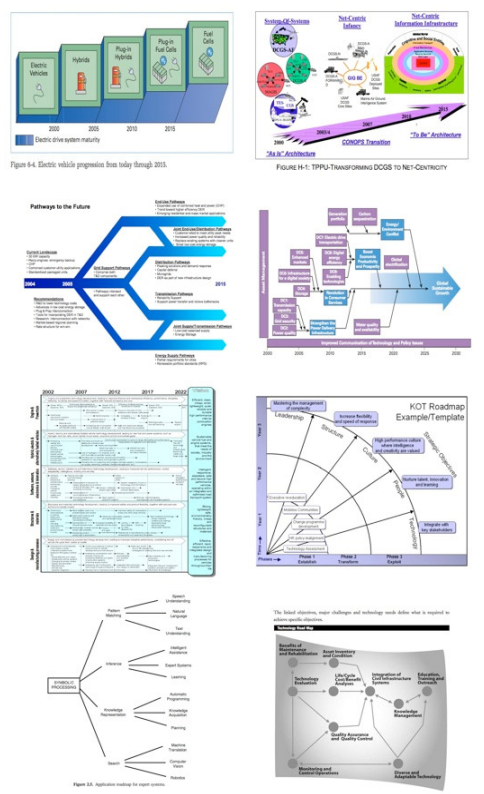
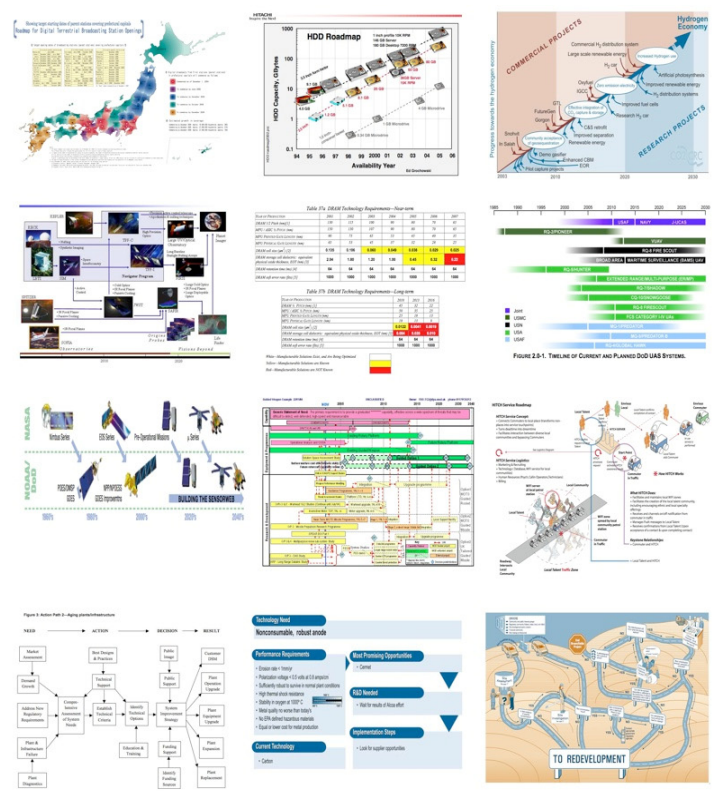

Fig. 2. Selection of visual roadmap representations, illustrating the variety of formats used

\section{Method}

\section{Participants}

The primary objective of the study was to define a typology of roadmap features with a view to characterising best-practice. However, roadmaps take many different forms and there is no authoritative definition with regard to which of these forms is more or less typical, or which of them should be regarded as "proper" members of the set. We therefore decided to proceed with our research on the basis that the set should be defined by consensus of utility to practitioners, through facilitated group meetings with expert informants. Unfortunately, the body of 'expert' practitioners for an evolving diagram class is not easy to define. Many diagrams in our sample might have been drawn by a corporate manager for whom this was the first and last roadmap they ever drew. It may be the case that common practices are emerging only through borrowing of features and slow evolution.

Our approach was therefore to treat aspirations of leadership within this community as being correlated with reflective professional practice. There are a small number of international consultants, academics and policy leaders who see roadmapping as being a central component of their professional skills. We invited a sample of these international leaders in strategic roadmapping, together with several information design specialists, to a one-day workshop held in conjunction with the July 2007 International Summit on Visualisation (Burkhard et. al. 2007). All 12 participants were 
sufficiently interested in the topic to travel to Zurich at their own expense, and we considered this to be evidence in itself that our informants were motivated to act as representatives of their nascent professional community. For our purposes, this sample, which might otherwise be criticized as biased or self-selected, is desirable because of the fact that it represents (self-identified) professional leadership.

\section{Procedure}

At the start of the day, the 12 participants entered a meeting room in which hard copies of the full research corpus (a total of 450 A5 colour copies) had been distributed over all surfaces of the room. Participants were initially formed into three groups of four, each of which selected a sample of 20 roadmaps from the corpus. Each group selected their sample with the objective to illustrate one of three themes designed to address likely practitioner concerns: 1) Purpose of the roadmap, 2) Good visual structure and 3) Design pitfalls. Groups were instructed to make their sample as varied as possible, within the constraint of illustrating the allocated theme. A duplicate set for each sample was then extracted from a second collection of the roadmaps, enabling participants to work in six (changing and balanced) pairs to undertake a series of card sorting activities (Rugg \& McGeorge, 2005). This approach was inspired by the classic card sort study by Lohse et. al. (1994), which produced a broad classification of visual representations. The objective of card sorting techniques is organise a set of objects in terms of relative similarity, based on classification decisions made by a number of participants. In our workshop, each informant spent about 30 minutes sorting each set of 20 roadmaps into categories of their own choosing, while their partner recorded those choices.

The 12 independent card sorts for each of the three thematic samples were then statistically combined using hierarchical cluster analysis. On the basis of the cluster analysis, dendrograms were constructed for each theme, with each leaf node of the dendrogram corresponding to one of the roadmap illustrations from the original sample. We then re-drew the dendrograms at large scale, so that the original A5 illustrations could be fixed to a wall-size depiction of the cluster analysis (as shown later in Figures 4 and 5). These large-scale illustrations were prepared during a lunch break, keynote address and social activity in the middle of the day.

The large-scale dendrograms were presented to our expert informants as a "manufactured consensus". We acknowledged that, just as the corpus itself was highly diverse, the developing status of the roadmapping community meant that expert opinion would be diverse rather than uniform. We therefore asked the informants to accept this "consensus" as being a basis for further collaboration, rather than attempting to modify it directly. Instead, we asked groups to work together to find the best possible labels for the branch points in the dendrogram, taking the clusters themselves as given. These negotiated labels form a key finding of the facilitated process. Finally, the resulting dendrograms were tested by using the emerging structure to support the rapid development of 'meta roadmaps' (roadmaps of roadmapping research). This was based on a discussion of purpose and good visual structure, taking into account the identified design pitfalls. The most preferred meta roadmap was then presented to other conference attendees (Burkhard et. al. 2007). 


\section{Pilot Study}

The workshop described above was both methodologically and logistically challenging. The complex and detailed procedure necessitated that it be preceded by a pilot study. In late 2006 a pilot workshop was conducted with 16 graduate students undergoing professional training in engineering design and manufacturing management. The pilot workshop was conducted on the same basis as the main study but differed in two main aspects. Firstly, in the pilot workshop, there were four themes rather than three because the theme that eventually became "design pitfalls" was initially divided into those pitfalls relating to visual structure and those that relate to conceptual purpose. After this caused confusion in the pilot study, the two pitfall types were later merged into a single theme. (For statistical reasons, this reduction in themes also enabled a reduction in the number of participants.) Secondly, the pilot study was divided into two sessions, with the cluster analysis and dendrogram production completed during a two-week break between sessions. This allowed us to explore alternative software packages and representation types, as well as defining the length of the break that would be needed to conduct the analysis during the main one-day workshop.

\section{Results}

Three kinds of result were achieved from the method described. The first was three hierarchical classifications that emerged from statistical cluster analysis of the sorting decisions. The second was the descriptive labels for each part of the classifications that were developed by the group through a facilitated discussion process. Our final objective was to present the resulting labeled classifications as decision trees or guides that drew on the three themes, thereby contributing to the design of roadmap diagrams that will a) serve an explicit purpose, b) employ proven graphic practices, and c) avoid common design pitfalls.

Statistical analysis used the Prodax data analysis and visualization tool (www.prodax.ch). The first step was to construct similarity matrices for each theme, in which the 20 roadmaps were arranged on horizontal and vertical axes. Each time a participant sorted two roadmaps in a single group, the number in the corresponding cell of the similarity matrix is increased by one. The similarity matrices of all participants can then be aggregated through cluster analysis. Normalised multi-dimensional scaling (NMDS) was used to transform the similarity ratings of the participants into a Euclidean distance model (Borg \& Groenen, 2005: 411) that shows relative similarity of the 20 roadmaps in that theme as relative distance within a $2 \mathrm{D}$ plane (roadmaps that are more similar are closer together), as in Figure 3. The reduction of the multidimensional data space into this 2D plane results in a degree of "stress", where similar items appear further apart than they should do. The number at the bottom right of the plot indicates the degree of stress for this theme. 


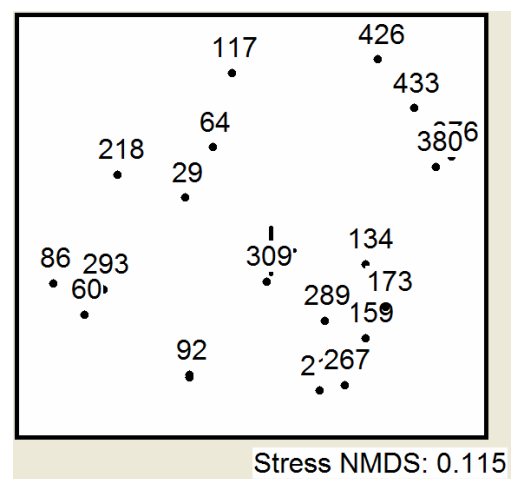

Fig. 3. Example result from multi-dimensional scaling analysis, showing roadmap similarity based on card sorts made within the purpose theme.

We also conducted an outlier analysis, through transformation of the similarity matrices, in order to check whether particular participants made sorting decisions that differed dramatically from all other participants. Figure 4 shows participant 33 as an outlier for the purpose theme. The results often corresponded to our own observations, with informants whose data appeared as an outlier also disagreeing during other workshop discussion (for example, that participant also appeared as an outlier in another theme). This is likely to be related to different professional or cultural perspectives of those informants, but we chose not to investigate the views of specific participants any further. Instead, we removed outlier sorting decisions when constructing the group consensus.
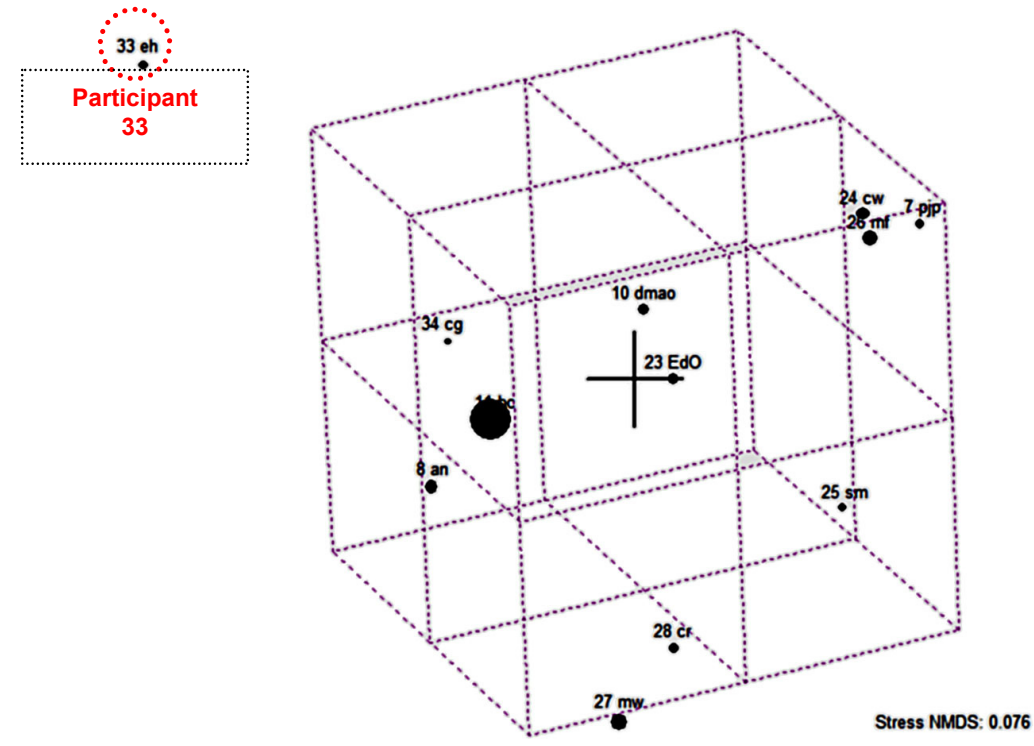

Fig. 4. Example of participant outlier analysis for the purpose theme. 
The average similarity judgments obtained using NMDS were then compared using hierarchical cluster analysis, producing a dendrogram for the purpose theme as shown in Figure 5a). This process starts with the two most similar items - in this case items 376 (partially covered) and 380 in Figure 3. The similarity measure indicates that these two items were grouped into the same class most frequently. These two are linked as the first "cluster" of two items at the left of Figure 5a). The analysis continues to group the most closely related items in order, combining these groups of two into higher level clusters, until the upper levels of the hierarchy show the most generic distinction between different types of roadmap.

\section{Purpose}

In the purpose theme, this highest level classification is between roadmaps made for specific scheduling of goal achievement (with an explicit time indication) and roadmaps designed for general orientation and visioning (without explicit time indications). Figure 5b) shows the large scale version of the dendrogram that was created during the workshop break, onto which the original A5 roadmaps were attached. The labels created by participants (written on Post-it notes) have been reproduced at the left, and the numerical identifiers corresponding to the statistical analysis are shown at the right.

\section{Good visual structure}

This process was repeated for the themes of good visual structure and design pitfalls. Figure 6a) shows the resulting dendrogram from hierarchical cluster analysis of the sample for the visual structure theme, and Figure 6b) the labels agreed for the higher level categories. This shows a distinction on the highest level among diagrammatic and pictorial roadmap formats. The roadmap formats perceived to be similar by the most participants are formats 363 and 14, both consisting of a table-like organization of information that was enhanced through the use of icons and imagery. Roadmap number 422, a Gantt chart-like format, achieved the least agreement regarding its grouping of all formats in this selection. This was reflected by the fact that, when labelling the resulting dendrogram, participants did not agree on a suitable label for the branch that contained this figure by itself. 


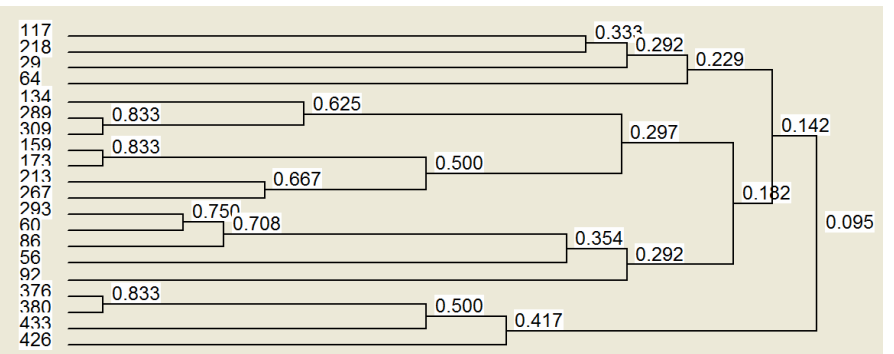

b)

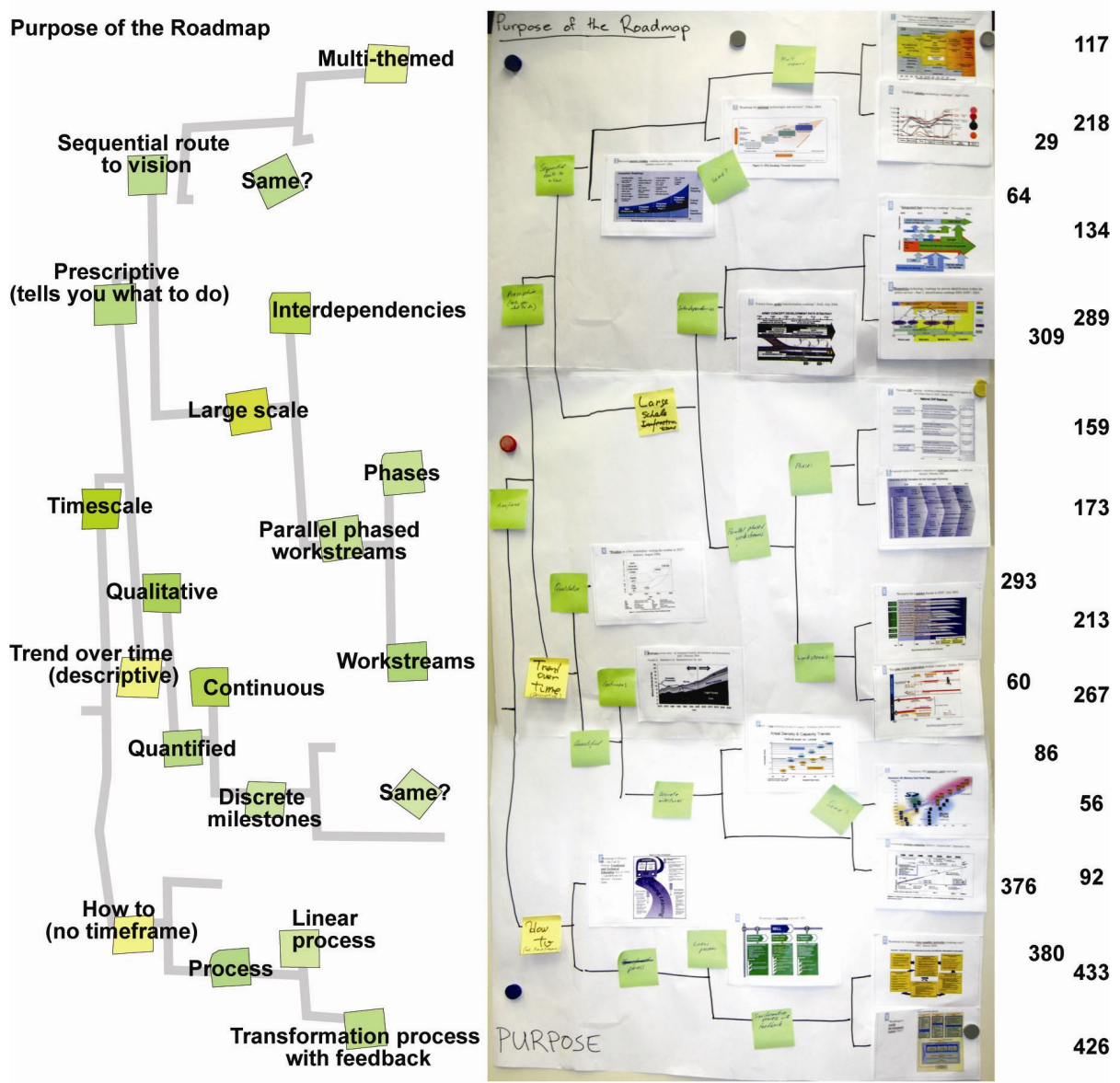

Fig. 5. Hierarchical cluster analysis results a) and resulting category labels b) of the dendrogram for the purpose-oriented classification of roadmap formats. Part b) includes a photograph of the large-scale chart created at the workshop. Note that the chart was reversed with respect to the cluster analysis, in order to emphasise categories. For clarity, labels have been reproduced in a larger font at the left of the photograph, and cluster analysis codes at the right. 
a)

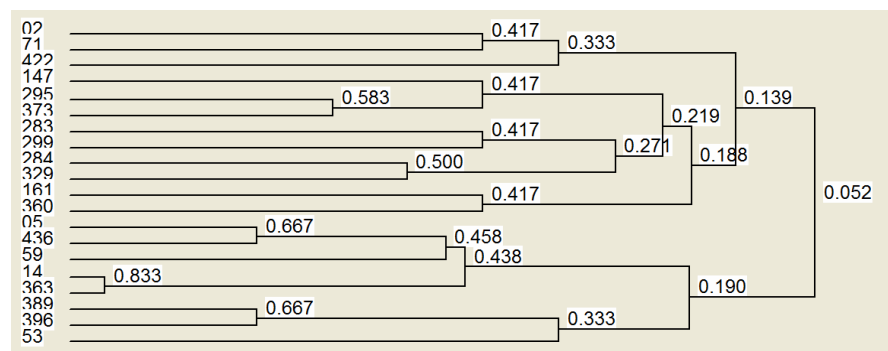

b)

Good Visual Structure
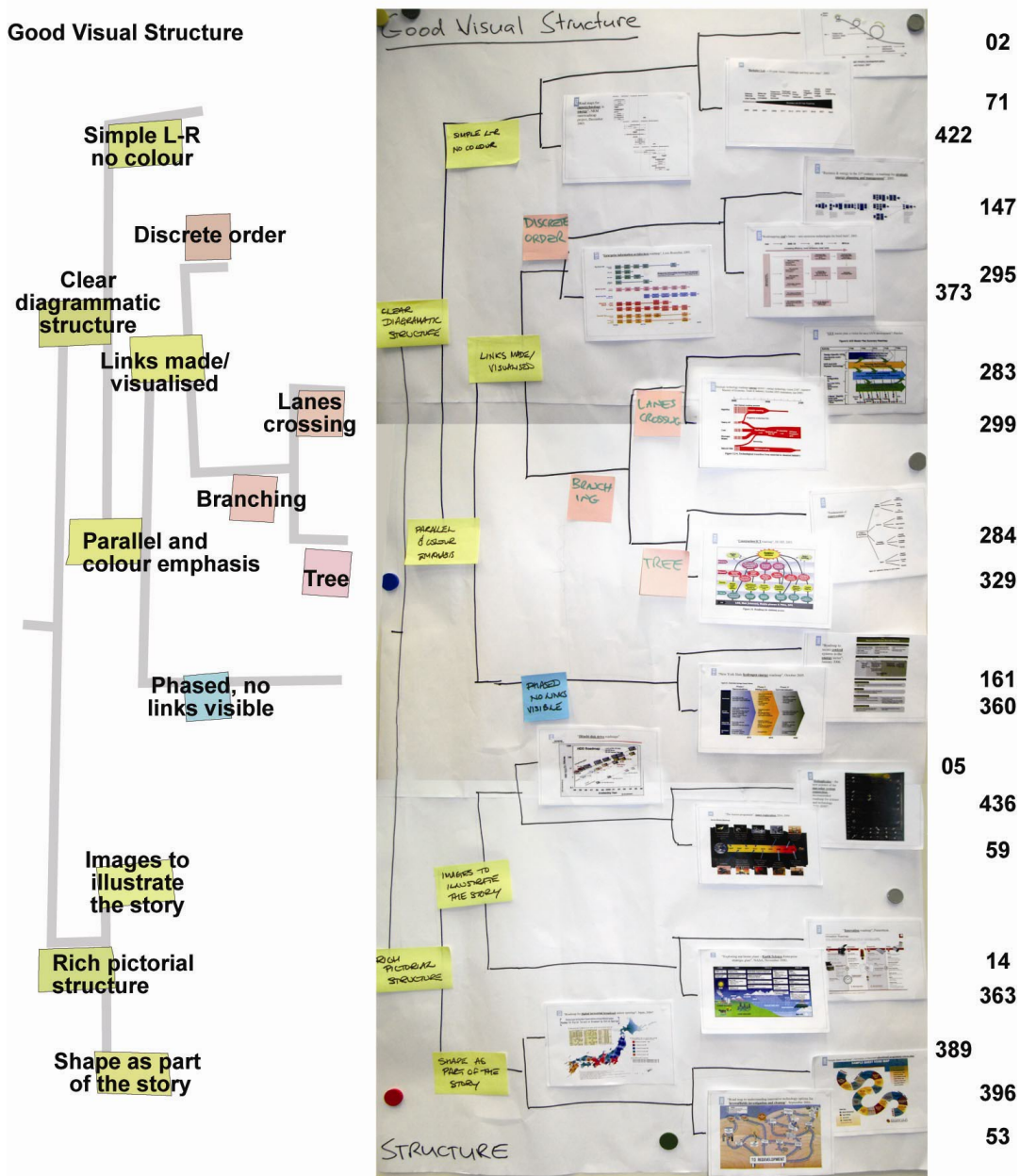

Fig. 6. Hierarchical cluster analysis results a) and resulting category labels b) of the dendrogram for the good visual structure classification of roadmap formats. As before, part b) shows the workshop chart, with labels repeated at the left and cluster analysis codes at the right. 


\section{Design pitfalls}

Regarding bad design practices the highest level distinction related to roadmaps that were either cluttered/overloaded (too much content) or failed to convey a consistent message because they lacked an organizing overall logic or scheme. The roadmap formats judged to be similar regarding their design mistakes by the most participants were two formats (337 and 339) that both contained more than 40 unstructured boxes with many connections and two formats (324 and 350) that did not contain any layers but more than ten related sections. We have not included the full set of classified design pitfalls in this paper due to space constraints, but the specific examples mentioned are illustrated in figure 7.

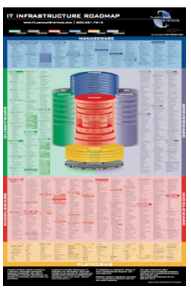

324

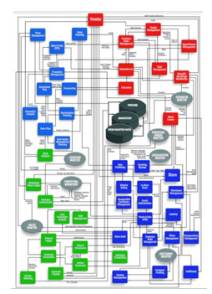

337

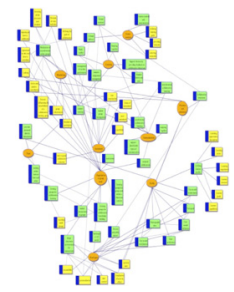

339

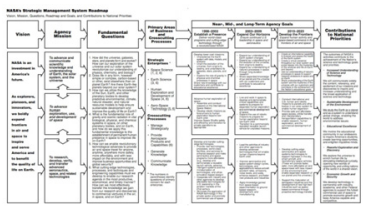

350

Fig. 7. Examples illustrating common categories of design pitfalls.

\section{Summary}

Overall, we found that dendrograms created using hierarchical cluster analysis provided a valuable tool to streamline group discussion on strategic purpose, and on the types of visual approach that could be used in order to achieve those purposes. Statistical cluster analysis created a manufactured consensus that could readily be interpreted by labelling top level categories. Close correlations at the bottom level of this hierarchy provided an opportunity for participants to recognise similarities among different members of an agreed common class. At intermediate levels, where atypical examples from the corpus were not strongly related to others in the sample, or where there was less consensus with regard to similarity, participants responded either by not labelling the branch, or by questioning whether these might be further examples of a higher level common category. In either case, we suggest that ability of expert participants to label the resulting categories is likely to be well correlated with the utility of those categories to practitioners.

Classification of common design flaws may be of less value for design guidance (specific examples demonstrate likely flaws better than category descriptions), but still provide benefits by prioritising the kinds of warning that might be given to practitioners who wish to avoid possible pitfalls. 

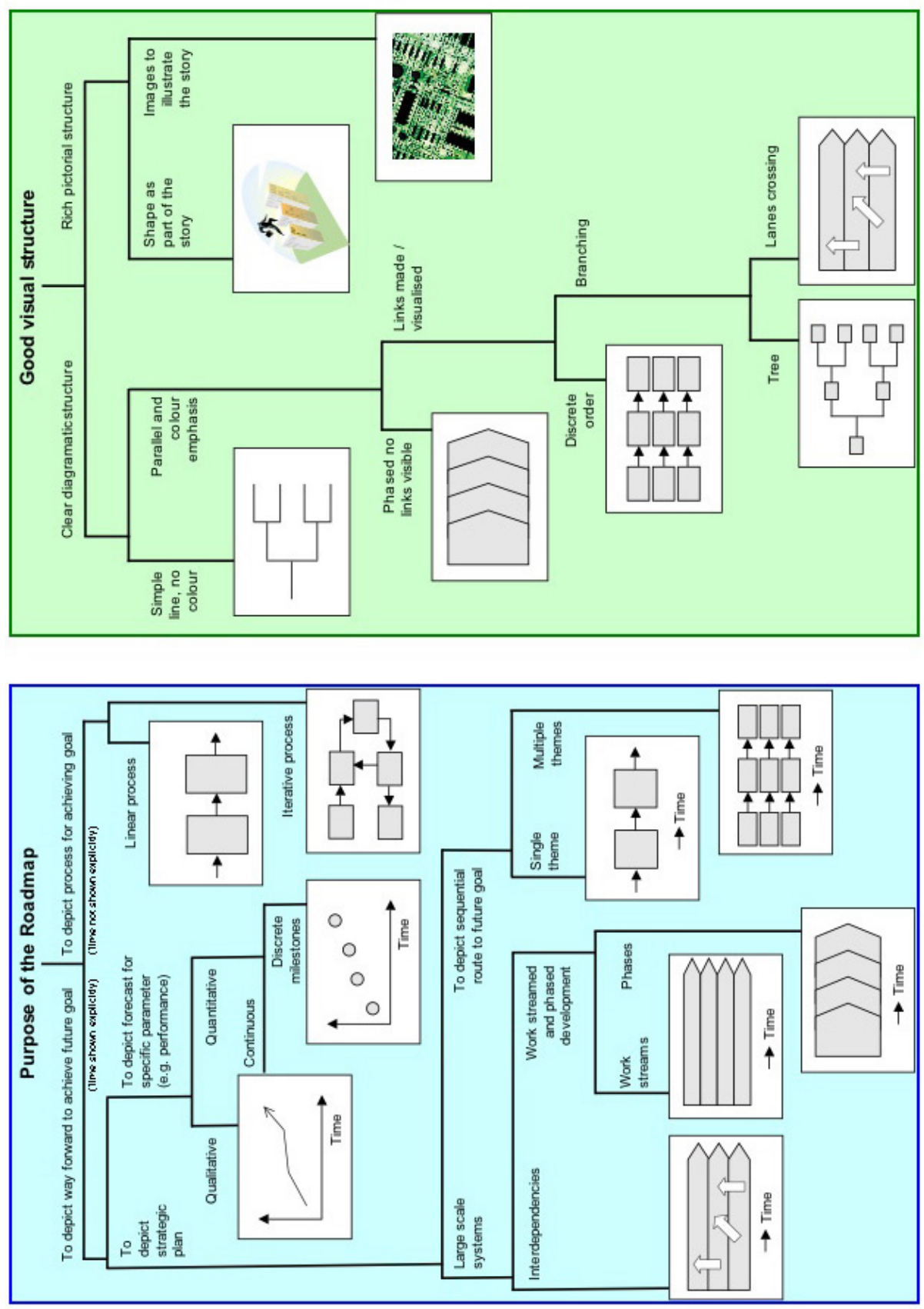

Fig. 8. Decision trees, based on Zurich workshop dendrograms, for use in a practitioner workshop. These illustrate decision trees for roadmap purpose and visual structure. 


\section{Practitioner evaluation}

An initial opportunity to test whether the outputs from the Zurich workshop are likely to be relevant to strategy practitioners was provided by an invitation to run a further workshop at an industrial symposium (the $13^{\text {th }}$ Cambridge Technology Management Symposium, in September 2007). Rather than repeating the card sorting exercise (which had been used both in the Zurich workshop, and in the earlier pilot), we chose to test the relevance of our results to practitioners by developing the dendrograms resulting from the Zurich workshop into pictorial decision trees (Fig. 8). These were then used as design frameworks for small groups of workshop participants to develop a roadmap for a strategic management topic of their own choice (Fig. 9)

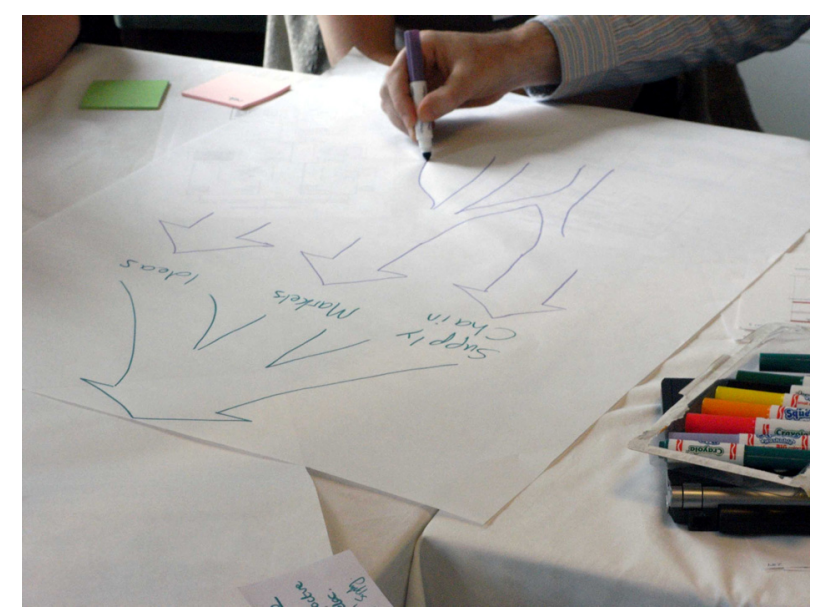

Fig. 9. Roadmap design activity at practitioner workshop, after choosing goal and structure from use of decision trees.

This practitioner event demonstrated the potential utility of the decision-tree roadmap design concept, although further work is needed to develop the decision tree and associated guidance relating to organizational function. Groups were able to design and construct a roadmap quickly (45 minutes). However, it was observed that groups containing participants with prior experience of roadmapping tended to use roadmap structures influenced by their previous experience (these were somewhat similar to the structure originally developed by Motorola).

\section{Discussion}

Roadmaps are a significant and widespread business tool. We wished to establish some consensus about their desirable visual features and potential applications, at a level that would provide a principled basis for design guidance. In much research into the properties of diagrams, it is hard to establish direct connections between application needs such as these, and available empirical research methods. However, we 
were successful in creating a summary description of a large and complex representative corpus, with structural categories created by, and recognizable to, domain experts.

In seeking to define normative standards for any type of diagram, there is an inherent trade-off between the desire to obtain objective, balanced guidance and the desire to exploit the personal, subjective judgements of experts who represent the views of the main stakeholders. Our workshops sought to resolve this conflict by applying an (objective) cluster analysis to the (subjective) judgements of relevant experts. As a result, the output from the workshop is a rigorously derived, inter-subjectively agreed, normative typology for roadmap diagrams.

This research approach offered a number of distinct benefits relating to the research corpus, the workshop participants and the selected methodology. Firstly, the unique corpus of material upon which the study was conducted is comprehensive, tangible and accessible. Secondly, the workshop participants represent experts from the various domains most relevant to roadmapping (including managers, visualisation scientists and information designers). Thirdly, the necessarily subjective activity of defining best-practice was tempered by employing the rational technique of cluster analysis to construct a consensus among the various experts. In combination, this approach allows a variety of perspectives to be brought to bear on a large sample of real-world material whilst retaining some objectivity and coherence.

Within the constraints of a workshop session, certain methodological limitations are unavoidable, and consideration must be given to any bias that these limitations impose. Firstly, there is the possibility that under time constraints, the participants involved in the selection and sorting of roadmaps (for 'goodness' or 'quality') will exhibit undue preference for overly simplistic or graphically seductive representations. Secondly, that by viewing these representations out of context, the roadmap sample must be judged with respect to the inferred objectives of the roadmap producers (including their intended communicative goal and the anticipated target audience). However by assembling a team of participants - expert in both the production and interpretation of roadmaps - efforts were made to mitigate these potential limitations.

We believe that this study has characterized the domain of strategy roadmaps sufficiently well to allow future investigation of specific applications and visual forms. One contribution from our research is therefore the creation of an initial exploratory structure of the domain. We have also demonstrated that even initial characterization provides a basis for some practical guidance to practitioners, by the simple strategy of turning a research categorization into a decision tree for initial stages of design. Finally, by addressing a context of diagram use that is quite distinctive, yet rather different in nature to more typical educational and technical uses of diagrams, we have found it necessary to develop a qualitative empirical methodology that is well suited to engaging with expert practitioner groups. We expect that this methodology will be of value in future studies of emerging diagram use in specific professional contexts. 


\section{Acknowledgements}

We thank the workshop participants: students of the EDC and IfM for their assistance in the first workshop, organizers and attendees at the Technology Management Symposium, and expert informants at the Zurich workshop: Brock Craft, Elke den Ouden, Mike Ferril, Clive Goodchild, Elizabeth Harvey, Ralph Lengler, Steve Mann, Andreas Neus, Dominic Oughton, Paul Palmer, Clive Richards, Masayoshi Wanatabe, Colin Winfield. Alan Blackwell's contribution to this project was sponsored by Boeing Corporation.

\section{References}

Bresciani, S., Blackwell, A.F. and Eppler, M. (2008a). A Collaborative Dimensions Framework: Understanding the mediating role of conceptual visualizations in collaborative knowledge work. Proc. 41st Hawaii International Conference on System Sciences (HICCS 08), pp. 180-189.

Bresciani, S., Blackwell, A.F. and Eppler, M. (2008b). Choosing visualisations for collaborative work and meetings: A guide to usability dimensions. Darwin College Research Reports DCRR-007, available online at www.dar.cam.ac.uk/dcrr/

Borg, I., Groenen, P.J.F. (2005). Modern Multidimensional Scaling, 2nd Edition, New York: Springer.

Burkhard, R.A., G. Andrienko, N. Andrienko, J. Dykes, A. Koutamanis, W. Kienreich, R. Phaal, A. Blackwell, M. Eppler, J. Huang, M. Meagher, A. Grün, S. Lang, D. Perrin, W. Weber, A. Vande Moere, B. Herr, K. Börner, J.-D. Fekete and D. Brodbeck (2007). Visualization Summit 2007: Ten research goals for 2010. Information Visualization (2007) 6, 169 188.

Cosner, R.R., Hynds, E.J., Fusfeld, A.R., Loweth, C.V., Sbouten, C. and Albright, . (2007), 'Integrating roadmapping into technical planning', Research Technology Management, Nov.Dec., 31-48.

de Laat, B. and McKibben, S. (2003), The effectiveness of technology roadmapping - building a strategic vision, Dutch Ministry of Economic Affairs, www.ez.nl

Lohse, G., Biolsi, K., Walker, N., Rueter, H. (1994) A classification of visual representations. Communications of the ACM 37(12), 36-49.

Phaal, R., Farrukh, C.J.P. and Probert, D.R. (2004), 'Customising roadmapping', Research Technology Management, 47 (2), 26-37.

Phaal, R. (2006). Technology and other (mostly sector-level) published roadmaps. Available online from www.ifm.eng.cam.ac.uk/ ctm/trm/documents/published_roadmaps.pdf

Rugg, G., Mc George, P. (2005) The sorting techniques: a tutorial paper on card sorts, picture sorts and item sorts, Expert Systems, 22 (8), 94-107.

Willyard, C.H. and McClees, C.W. (1987). Motorola's technology roadmap process. Research Management, Sept.-Oct., pp. 13-19. 